\title{
Traduire
}

Revue française de la traduction

\section{À la courbe des eaux, ou traduire le théâtre coréen aujourd'hui en France}

Yumi Han

\section{(2) OpenEdition}

Journals

Édition électronique

URL : http://journals.openedition.org/traduire/444

DOI : 10.4000/traduire.444

ISSN : 2272-9992

Éditeur

Société française des traducteurs

Édition imprimée

Date de publication : 15 juin 2010

Pagination : 73-86

ISSN : 0395-773X

\section{Référence électronique}

Yumi Han, «À la courbe des eaux, ou traduire le théâtre coréen aujourd'hui en France », Traduire [En

ligne], 222 | 2010, mis en ligne le 12 novembre 2013, consulté le 01 mai 2019. URL : http://

journals.openedition.org/traduire/444; DOI : 10.4000/traduire.444 


\section{À la courbe des eaux, ou traduire le théâtre coréen aujourd'hui en France}

HAN Yumi

\section{Introduction}

Traduire, surtitrer, éditer le théâtre coréen : si nous avons la chance de pouvoir occuper les trois positions selon les circonstances, il est certain que le geste traducteur est primordial, et nous sommes très heureux de pouvoir partager un peu de cette expérience dans l'espace confraternel que nous offre la revue Traduire(1). Cela va être l'occasion de présenter succinctement la caractéristique actuelle de la réception, en France, aujourd'hui, des arts de la scène coréens, partagés entre un théâtre contemporain inconnu et un art traditionnel classé comme musical, que nous considérons comme les deux faces d'un même art identitaire, à faire découvrir comme tel. Nous verrons alors dans quel cadre général de traduction littéraire nous tentons de nous situer, afin de pouvoir nous adonner pleinement à la spécificité de l'écriture théâtrale : comment ne pas faire perdre le souffle au lointain texte d'origine lors de son voyage vers les Français, comment le "mettre en scène " au mieux dans sa langue d'arrivée et l'offrir en partage?

\section{État des lieux}

La grande force du traducteur de théâtre est - idéalement - de pouvoir couvrir l'ensemble du champ s'étendant de l'écriture du texte à la vérification de sa juste oralité, de la solitude de la page au travail en équipe de la scène, et inversement, dans le but de peaufiner et d'enrichir la traduction. Comment ne pas avoir envie, comme André Markowicz, de retraduire les textes à chaque nouvelle reprise, ou de débattre, comme J.-M. Déprats, des problèmes posés par la

(1) Le "nous " utilisé dans cette article n'est pas que de " majesté " : il désigne le binôme de traducteurs, surtitreurs et directeurs de collection, que nous formons depuis quinze ans avec l'écrivain Hervé Péjaudier, à l'article duquel je renvoie dans ce même numéro, en particulier sur la question cruciale du surtitrage, que je ne traite donc pas ici. 
prolifération de traductions scéniques au coup par coup ? Hélas, l'état de reconnaissance du théâtre coréen en France aujourd'hui est encore bien insignifiant, et nul Shakespeare, nul Tchekhov, n'y existe comme tel. Cela ne change pas la nécessité d'une prise en compte des spécificités scéniques des textes, mais nous sommes bien obligés de prendre aussi en compte la réalité culturelle et économique présente, à savoir la méconnaissance profonde de ce théâtre en France. D'un autre côté, cela offre le charme des explorations pionnières, lorsque tout est en friche et que le moindre bourgeon semble promettre mille printemps parfumés(2)... Néanmoins, si l'on veut réfléchir à la situation de notre travail, il est nécessaire de voir comment il s'inscrit dans un état des lieux plus global.

La traduction littéraire d'œuvres coréennes est assez récente, et sa reconnaissance publique ne date que de la fin des années 80 , avec la première création d'une collection spécifique chez un grand éditeur(3). Durant les années 90 , la découverte en Occident de la "nouvelle vague " de cinéma coréen a permis à ce mouvement de se développer, et de nouveaux éditeurs se sont intéressés à la Corée, ponctuellement ou durablement, mais presque toujours exclusivement autour du roman contemporain. En 2004, nous avons eu la chance de pouvoir fonder une collection a priori destinée aux arts de la scène : "Scènes Coréennes ", aux éditions Imago, dont nous saluons ici l'engagement. On voit à la fois combien l'arrivée de la littérature coréenne sur le marché français est extrêmement récente, mais aussi combien la situation avance à grands pas(4). L'histoire de la traduction du coréen en France semble donc se normaliser, avec un étiquetage réservé sur les rayons des libraires, même si cet espace est encore bien restreint, écrasé entre les imposants voisins "Chine " et " Japon ".

II existe pourtant une vraie particularité de la traduction du coréen, qui, me semble-t-il, est une donnée historique : la quasi-totalité des traducteurs du coréen fonctionnent aujourd'hui en binôme franco-coréen. C'est certainement un phénomène transitoire, lié au trop petit nombre de bilingues littéraires et à la demande expresse des Fondations. Cela a au moins permis d'échapper aux terribles " traductions de la traduction anglaise ", et le développement, lent mais régulier, des études coréennes en France voit émerger une toute nouvelle génération de traducteurs " solo ". Cependant, je voudrais m'attarder quelques instants sur cette situation singulière, souvent décriée comme un pis aller, mais qui se révèle aujourd'hui, bien comprise, comme un véritable atout, spécialement lorsqu'il s'agit de théâtre, ou plus généralement d'oralité du texte (mais y a-t-il des textes littéraires qui échappent à l'épreuve de la voix ?) Oublions

(2) Saluons au passage Cathy Rapin et Im Hye-Gyeong pour le travail de défricheuses qu'elles accomplissent depuis de nombreuses années à Séoul, cf. bibliographie.

(3) Nous ne voudrions pourtant pas oublier les aventureux pionniers que furent dès les années 60 des enseignants comme Marc Orange ou Roger Leverrier, entre autres.

(4) II faut bien sûr souligner ici l'importance de l'engagement de l'État coréen, qui depuis plusieurs décennies aide la traduction et l'édition dans les principaux pays européens, par le biais de Fondations publiques ou privées sans l'aide desquelles la situation serait très différente. Saluons aussi le travail important de relais joué par le Centre culturel Coréen de Paris. 


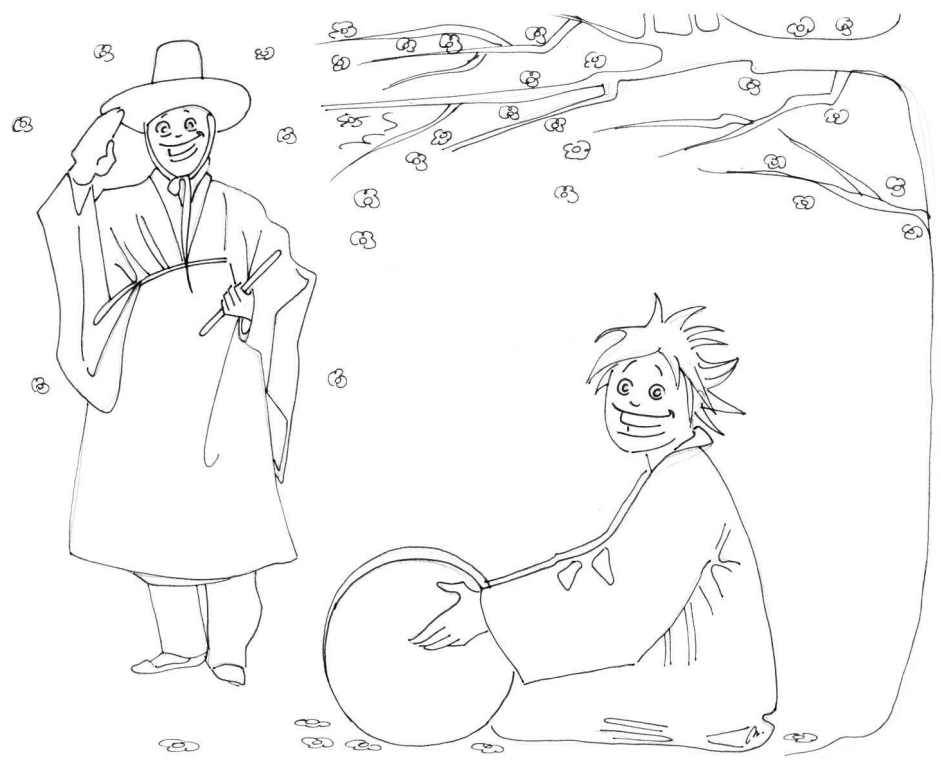

Illustration : Marlène Junius, http://alotoftralala.over-blog.com

le cliché selon lequel le traducteur coréen apporterait un texte " mot à mot " à son partenaire chargé de le "mettre en bon français ", tant ces notions relèvent d'époques révolues et n'ont aucun sens en traductologie. II s'agit d'un véritable travail de collaboration, à l'image de cette tentative réussie par les éditions Bayard dans les années 2000 de réaliser une nouvelle traduction de la Bible en associant, livre par livre, un poète et un théologien. Toute proportion gardée, on voit ici le mécanisme de traduction idéalement à l'œuvre dans un binôme, la partie coréenne étant garante du message d'origine, au sens large, c'est-à-dire de son immersion dans une culture donnée, la partie française ayant en charge la " mise en scène " du texte dans la langue d'arrivée, sa mise en tension dans la culture française, sa dramaturgie. La collaboration serrée entre les deux permet ainsi de mettre en œuvre un processus essentiel d'interculturalité, un questionnement qui touche tous les registres de la traduction. II nous permet également de tester à l'oreille, simultanément, les effets produits entre les deux langues, à haute voix. Lors de la traduction de Muldoridong, ce "village à la courbe des eaux ", en particulier, il y avait de nombreuses " chansons ", relevant à la fois du chant ancestral chamanique et de la poésie moderne, qui demandaient un traitement particulier, et c'est vraiment l'oreille intérieure qui a validé nos choix : aucun des deux n'étant en garde plus particulièrement du sens ou du son, c'est ensemble que les traducteurs vont faire résonner les deux cultures entre elles.

Une autre spécificité de la culture coréenne en France aujourd'hui est sa présence simultanée sous deux aspects très distincts, d'un côté une tradition " immémoriale ", et de l'autre une modernité 
de plus en plus " en pointe ". Les traductions modernes ont d'emblée concerné des romanciers contemporains se confrontant à l'histoire tragique de leur pays, et aujourd'hui on sent les éditeurs à la recherche d'auteurs plus jeunes, plus provocants dans leur approche d'une Corée postmoderne, en liaison avec un cinéma qui jouit d'une reconnaissance internationale. De l'autre côté, les festivals s'intéressent à ces genres scéniques traditionnels que sont le pansori (forme de récit chanté pour une voix et un tambour datant du XvIIIe siècle et dont cinq pièces nous sont parvenues par transmission orale) ou les kut (rituels chamaniques ayant assez bien traversé l'épreuve des siècles). Le point culminant de cet intérêt a eu lieu au Festival d'Automne à Paris en 2002, avec un panorama de tous les arts de la scène traditionnels coréens, intégrale surtitrée des pansoris, théâtre masqué, rituel chamanique ou danse de cour, et une section " cinéma d'aujourd'hui ". Mais il n'y avait eu aucune place pour le théâtre contemporain, considéré comme "trop éloigné des standards internationaux ". Voilà donc bien le hiatus auquel nous sommes confrontés : le théâtre coréen, s'il est contemporain, n'est pas digne d'intérêt parce que ses mises en scène ne sont pas " au niveau ", et s'il est traditionnel, il est alors considéré comme un spectacle musical (le modèle étant le pansori, défini comme une forme d'opéra à une voix) et ethnique, en particulier dans le cas du rituel chamanique. Sans doute s'agit-il des contrecoups du mouvement de patrimonialisation lancé par le gouvernement coréen dès les années 60 au niveau national, puis international, avec la reconnaissance du pansori comme patrimoine immatériel en 2003 par l'Unesco(5), mouvement qui comprend toujours le risque de muséifier des genres en danger. Toujours est-il que le Festival d'Automne avait choisi de ne pas sacrifier la dimension scénique du pansori, c'est-à-dire d'un récit durant plusieurs heures, en choisissant de présenter des intégrales, ce qui ne se faisait presque jamais à l'étranger, et qui imposait le surtitrage : et voilà comment nous nous sommes retrouvés en charge de l'intégrale des pansoris, vingt heures sur cinq soirées.

Cette leçon fut inoubliable, et son succès auprès du public français, mais aussi coréen, nous a fait sentir à quel point la tradition coréenne opère la fusion entre le texte, le chant et le geste. On trouve aussi le contact permanent avec un public participant, sans compter, avec le rituel chamanique, la musique instrumentale, les décors flamboyants et les effets spectaculaires. Nous avons alors mieux compris d'où provenait la problématique des metteurs en scène coréens. Lorsque Lee Jong-il était venu en 2000 à Paris avec la pièce Tertre, du poète dramaturge 0 Tae-sòk, beaucoup de spectateurs avaient été un peu gênés par un aspect dit seventies du travail, qu'ils jugeaient daté, tandis que de notre côté, soucieux de respecter la scénographie chorale, nous avions réduit au plus simple le surtitrage, du coup trop succinct(6). Mais lorsqu'il est revenu en 2004 présenter Muldoridong, de Hò Kyu, autre pièce moderne,

(5) Sur cette question, cf. HAN Yumi, "La patrimonialisation du pansori, art de la scène coréen, et son impact sur l'évolution du genre ", intervention aux Journées d'études "Formes spectaculaires traditionnelles et processus de patrimonialisation ", 25-26 mars 2010, Université européenne de Bretagne, Rennes, Actes à paraître.

(6) Cf. l'article d'Hervé Péjaudier dans ce même numéro. 
mise en scène selon les mêmes principes, nous avons compris quelque chose concernant un possible malentendu. En effet, que raconte cette pièce ? Dans ce village à la courbe des eaux, (mul, eau, dori, courbe, dong, village), rien ne va plus depuis que la fiancée d'une âme morte aime le Gars, qui, pour son châtiment, devra fabriquer des masques rituels chargés de sauver un ordre ancestral tyrannique, ce qu'il fera au sacrifice de sa vie. La pièce est un long poème dramatique, scandé de chants de type chamanique, mais aussi de scènes burlesques masquées, proches de notre commedia dell'arte. On voit que cette pièce, qui date des années 70, s'inscrit dans le mouvement mondial d'un théâtre de contestation qui s'attaque aussi aux formes considérées comme bourgeoises et réactionnaires du théâtre dominant, en renouant avec des traditions plus archaïques, à la recherche d'une refondation de la scène par le collectif, le corps, la poésie. II se réclame d'un art total destiné à exprimer la révolte de la jeune génération, opposant au matérialisme égoïste les besoins d'une spiritualité généreuse. La jeunesse coréenne n'échappait pas à cette vague de fond, mais, peut-être à la différence d'une jeunesse occidentale cherchant à retrouver des traditions disparues, ils avaient les traditions chamaniques sous les yeux, toujours vivantes, ainsi que les traditions populaires musicales, chorégraphiques...

II faut se souvenir que la Corée des années soixante, du moins la partie de la péninsule dite du Sud, est l'héritière de la dynastie Chosòn, fermée à la modernité jusqu'au début xxe, colonisée par le Japon impérial jusqu'en 1945, déchirée par une guerre civile (1950-1953), pays du tiers monde sous dictature militaire et protection américaine... Les auteurs de théâtre autre que de divertissement sont rares, et écrivent sous l'influence occidentale masquée d'auteurs de gauche comme Salacrou, pour 0 Young-jin(7), ou de Brecht, communiste, donc interdit, pour Lee Gun-sam(8). Ce dernier sera le professeur de toute cette jeune génération dont nous parlons et qui fera exploser les carcans occidentaux en renouant avec les techniques de jeu traditionnelles. Paradoxalement, la jonction entre le théâtre moderne et les arts traditionnels a pu s'opérer grâce au travail de patrimonialisation opéré dès le milieu des années 60 par le gouvernement, qui a commencé à faire l'inventaire du patrimoine de cette Corée désormais du Sud, et particulièrement du patrimoine dit « immatériel ", distribuant des titres de Trésor National Vivant, assignant aux Détenteurs d'un chant, d'une danse, d'un rituel, la mission de transmettre leurs connaissances. C'est ainsi que se sont croisés des jeunes gens d'horizons variés, unis par la passion des arts de la scène et l'envie ardente de résister à la muséification nationaliste, et que des troupes se sont constituées pour inventer un nouveau théâtre à la fois viscéralement coréen, et populaire, donc ouvert au monde. "Et plus ce sera coréen, plus ce

(7) Cf. 0 Young-jin, 2000.

(8) Cf. Lee Gun-sam, 2010, en particulier la postface de Choe Junho qui explique bien les enjeux de cette époque. Pour une approche plus globale de l'histoire du théâtre coréen, on lira avec profit l'article de Choe Junho 2005. 
sera universel ", comme l'écrivait Hò Kyu, grande figure parmi ces poètes, chefs de troupe, directeurs de théâtre, et dont les textes, trente ans plus tard, n'ont rien perdu de leur force(9). Du coup, notre conception s'est modifiée, et nous avons choisi de réaliser un surtitrage intégral, au plus près du texte, qui tente de rendre compte de ses qualités poétiques et prosodiques. Nous nous sommes alors convaincus de la similitude de fond qui existait entre le pansori et ce théâtre moderne coréen(10). Bien sûr, les deux genres sont historiquement distincts, mais leur problématique peut se considérer aujourd'hui de manière similaire. II s'agit dans les deux cas de textes qui ont une vraie force autonome à la lecture, mais qui, dans les deux cas, attendent la réalisation scénique pour prendre leur ampleur, ce qui donne au traducteur la responsabilité supplémentaire de ne pas escamoter cette dimension.

\section{Traduire}

La confrontation de nos traductions avec les contraintes du surtitrage et de l'édition a ouvert notre approche de traducteurs, en nous confrontant à la pratique de la scène, au travail d'équipe, à la question de la réception. Toujours est-il que le traducteur, même dédoublé, se retrouve toujours seul devant sa page, blanche comme la nappe de la fameuse table d'hôte d'Antoine Berman... Et si, à chaque nouveau texte, le traducteur se retrouve obligé de négocier ses choix pragmatiques, nous pensons utile de préciser ici quelques règles de base qui guident notre réflexion dans ce domaine.

En nous appuyant sur la théorie interprétative de l'École de Paris(11), nous admettrons d'abord que pour traduire, il faut toujours interpréter. La dynamique dégagée, en trois étapes, nous semble particulièrement intéressante : d'abord la " compréhension " du texte de départ, suivie d'une "déverbalisation " avant la "réexpression ", qui donne le texte d'arrivée. La compréhension du texte est décrite comme " un processus qui dégage le sens d'une chaîne sonore ou graphique grâce à l'association des significations linguistiques et de compléments cognitifs " (Lederer 1994). D'emblée, on relie la connaissance linguistique et la connaissance extralinguistique, le notionnel et l'émotionnel, au sein d'un bagage cognitif. Sont mobilisés non seule-

(9) Cf. par exemple Roh Kyeong-shik, Un pays aussi lointain que le ciel, sur les familles séparées, dans un style proche du Théâtre du Quotidien, (Roh, 2004), ou à l'opposé, dans un genre surréaliste provocateur, Yi Hyòn-hwa, Qui êtes-vous ? (traduction Im-Rapin, collection Scènes Coréennes, éd. Imago, paru en juin 2010).

(10) Nous avons d'ailleurs eu une confirmation récente du lien qui unit le pansori au théâtre moderne, avec le succès en Corée du Dit du Si-tchuan, adaptation de La bonne âme du Si-tchuan de Brecht, coréanisée dans le style du pansoripar une fameuse chanteuse spécialiste de ce genre, qui a décidé de le revivifier à sa manière pour toucher un public plus jeune. Nous sommes très heureux de l'accueillir en France en mars 2011, en traduisant et surtitrant son texte.

(11) Nous renvoyons ici aux travaux de M. Lederer et D. Seleskovitch, de l'ESIT (École Supérieure d'Interprètes et de Traducteurs), dont le corps de doctrine a pris aussi le nom d'École de Paris. 
ment la connaissance grammaticale, morpho-syntaxique, de la langue du texte de départ, mais aussi un ensemble de représentations socioculturelles et historiques qui sont à l'œuvre au cœur du texte à traduire en une autre langue. Alors survient le temps de la déverbalisation(12), une sorte de "précipité chimique " où la grande complexité des données (linguistiques et extralinguistiques) de la première étape va se fondre comme dans un creuset, le résultat de cette opération permettant d'arriver à la réexpression. L'important, au cours de cette phase, est de parvenir à se détacher de la langue de départ pour accéder à une compréhension vraiment globale, nécessaire à l'établissement du texte d'arrivée. Au moment de la "réexpression ", et particulièrement si les deux langues se trouvent historiquement, géographiquement, anthropologiquement, éloignées, on voit bien comment le geste de traduction s'inscrit dans tout un contexte culturel, confronté à la manière dont une civilisation marque la langue de son empreinte. II s'agit d'affiner une compétence interculturelle qui permettra de faire le lien entre les deux cultures, et d'opérer des choix pour favoriser la réception de cette culture dans l'autre langue à un moment donné.

Toutes les remarques précédentes, théoriques et générales, ne prennent sens que lorsqu'elles se confrontent au contexte historique de la relation à un moment donné entre les deux langues concernées, c'est-à-dire entre les deux cultures. Pour prendre des exemples très simples, voyons les mots(13) kisaeng, hanbok, soju ou kimchi, qui désignent une courtisane, une robe, un alcool, et un plat de choux, tous "typiquement coréens ". Quand il s'agit de lexiques culturels dont le référent est pensé comme vacant(14) dans l'autre langue, quelle est la solution? Soit on élimine la question, en se rabattant purement et simplement sur les mots équivalents (une courtisane, une robe, un alcool, et un plat de choux). À l'autre extrême, on peut choisir d'emprunter tel quel le mot, et, selon le niveau de reconnaissance moyenne admissible supposé du lecteur, on l'explicitera plus ou moins, soit dans le corps du texte, soit dans une note, avec une certaine marge de variation selon le contexte ; ainsi, on peut avoir une kisaeng courtisane, un hanbok longue robe traditionnelle ou ensemble jupe boléro, etc. Selon la tentation encyclopédique du traducteur, la note pourra être plus ou moins développée, le soju étant désigné comme " alcool ", ou " alcool traditionnel à base de patate douce ou de riz titrant environ vingt degrés ", ou, pourquoi pas, " ce que boivent les personnages des films coréens". Bref, on voit que la marge est grande, à condition de ne pas confondre le corps du texte et les

(12) " II s'agit d'un affranchissement des signes linguistiques concomitant à la saisie d'un sens cognitif et affectif ", Lederer 1994, p. 213.

(13) Nous parlerons de mots " culturels ", de ceux qui, traduits, peinent à trouver les référents correspondants ou bien ne contiennent plus les mêmes valeurs d'origine.

(14) Nous insistons sur cette notion, pour mettre en garde contre l'annexionnisme réducteur, particulièrement dangereux lorsqu'il s'agit de transposer des réalités surtout politiques ou religieuses, avec le risque toujours présent de christianiser le bouddhisme, de " Mircea-Eliadiser " le chamanisme, d'occidentaliser les rapports de classes, etc. 
notes. Et de ne pas confondre non plus les cultures : revenons un instant sur notre exemple, pour démontrer assez simplement à quel point il s'agit d'une question historique d'assimilation. Si vous tapez sur votre ordinateur la phrase suivante : " la kisaeng en hanbok sert du soju et du kimchi ", ces quatre termes coréens très courants (en coréen) apparaîtront soulignés en rouge, comme mots fautifs. Mais si vous transposez la phrase comme suit : "La geisha en kimono sert du saké avec des sushi ", rien n'est souligné, le logiciel reconnaît tous les mots. Cet exemple exprime bien le décalage temporel de reconnaissance, en Occident, des cultures japonaises, puis coréennes. Que tout cela soit lié à l'histoire des civilisations est une évidence. C'est ainsi que, s'il y a quinze ans, on pouvait encore trouver " kimono coréen " pour hanbok, ou " soju, sorte de sake coréen ", nous avons découvert récemment dans la rubrique Cinéma du Monde le mot soju cité sans italique, ni notes, ni explicitation. La culture coréenne conquiert peu à peu son indépendance... Parallèlement, on commence à trouver dans les pages " économie " le mot chaebòl sans explicitation(15). II y va en quelque sorte de la responsabilité des traducteurs, et ce travail de passeur consiste aussi à importer, comme en contrebande, un certain nombre de mots considérés comme clés, que nous proposons comme emprunts possibles. L'Histoire choisira(16).

Quand on traduit, ce ne sont pas tant les mots ni les phrases qui sont l'objet de la traduction, mais un discours, qui est à la fois un ensemble d'énoncés résultant de l'acte d'énonciation, et le langage mis en action pour constituer une suite de messages ayant un début et une fin. Autrement dit, selon les termes d'Antoine Culioli dans sa théorie de l'énonciation, il s'agit de faisceaux de propriétés physico-culturelles, qu'il appelle des "Notions "(17). De fait, traduire une œuvre, c'est traduire les Notions qui se présentent dans cette œuvre, mais aussi considérer l'œuvre entière comme étant elle-même une "Notion " au sens de Culioli, c'est-à-dire un bloc de langage en évolution, englobant son propre univers, et transportant une vision du monde qui lui est propre.

Le lecteur d'une œuvre traduite devra donc lui aussi faire ce parcours à son niveau, et construire peu à peu ses propres "Notions ", aidé ou non par les notes, les glossaires, mais aussi par les préfaces, les quatrièmes de couverture, etc., et tout ce que Gérard Genette étudie sous le nom de paratexte. Ces "Notions" en acte dans la traduction qu'il lit, il est d'autant plus important que le lecteur y accède qu'il est confronté à un dédoublement bien connu, qui distingue la vision du monde d'une époque de celle d'un auteur, construite contre elle, comme en combat singulier. On voit la responsabilité des traducteurs (et des éditeurs) dans ce travail, qui,

(15) Ce qui bien sûr ne préjuge en rien de la compréhension réelle de cette notion par le lecteur français, mais c'est une autre histoire (le lecteur est-il beaucoup plus au fait de ce que recouvre le terme bien français de " conglomérat "?)

(16) Sur ces questions " d'étrangeté ", cf. Han-Péjaudier 2007.

(17) Culioli A. (1983-1984), Séminaire de D.E.A., U.F.R. Université Paris 7. 
en plus, comme tout bon travail, ne doit pas être un pensum. II ne s'agit ni de conforter le lecteur dans ce qu'il connaît, ni de lui infliger une leçon, mais de l'inciter à une découverte active. Et, symétriquement, il s'agira de recevoir l'auteur en lui faisant une place à la grande table d'hôte dont parle Antoine Berman(18), dans le respect des lois de "l'Hospitalité langagière " telle que Paul Ricœur en a exposé les règles(19), avec la " complicité passionnée " et " l'aptitude à négocier à chaque instant la solution qui nous semble la plus juste " que recommande Umberto Eco(20).

\section{Théâtre}

Y a-t-il une spécificité de la traduction théâtrale, qui la distingue des problématiques de la traduction littéraire dans son ensemble ? Bien sûr, il y a une insistance marquée pour l'oralité, et l'on est confronté à la nécessité pour une pièce d'être jouée, c'est-à-dire proférée (même intérieurement, par un lecteur). Mais s'il est vrai qu'au théâtre, l'oralité est omniprésente, le problème est le même dans les œuvres non théâtrales laissant sa place à l'oralité, poésie, ou scènes dialoguées dans les romans. Et une pratique du théâtre n'est pas inutile pour trouver la juste oralité d'un poète, ou pour ne pas rendre trop ridicules les dialogues dans un roman réaliste - vrai souci en traduction, et dans n'importe quelle langue. Mais là où la question de l'oralité devient plus spécifique lorsqu'on traduit du théâtre, c'est dans le jeu permanent du texte et du sous-texte qui relient les répliques pour construire une continuité. Même si les didascalies servent au comédien ou au lecteur (et au traducteur !), une fois sur scène, tout se passe dans l'enchaînement des répliques entre des personnages. II faut savoir lire ce qui se passe entre les répliques, et le rendre palpable. L'épreuve de l'oralité est donc particulièrement nécessaire à l'élaboration d'une traduction théâtrale, même si, en ce qui concerne le théâtre coréen, les occasions sont encore plus que rares... Mais pour le peu que nous en avons vu, qu'il s'agisse de lectures ou de mises en scène, il est évident que cette épreuve du feu est essentielle, non à la conception d'ensemble, mais au polissage du texte, à la validation d'un certain nombre de choix, mais aussi à leur assouplissement, à leur ajustement. Sans remettre en cause les principes traductologiques adoptés, et dans leur esprit même, tel que nous l'avons défini ci-dessus, cette confrontation permet de poursuivre un travail sur-mesure très important. Par exemple, nous venons de présenter, en lecture mise en espace, quelques extraits d'une pièce de 1966 de Lee Gun-sam(21), initiateur du brechtisme en Corée, dans laquelle le personnage principal intercale entre chaque scène un monologue adressé au public : cela a permis

\footnotetext{
(18) Antoine Berman, 1984.

(19) Paul Ricœur, 2004.

(20) Umberto Eco 2007, p. 435.

(21) À la soupe ! , op. cit., présentation au Centre Culturel Coréen, Paris, le 27 avril 2010.
} 
un travail très intéressant avec le comédien pour savoir jusqu'où on pouvait pousser le texte d'origine, dans la distanciation et le rapport au public. Lors du débat qui a suivi, I'historien du théâtre Choe Junho a rappelé qu'à l'époque, ces parties étaient des canevas sur lequel le comédien brodait par rapport à l'actualité, l'essentiel étant d'amener le spectateur à réagir face aux situations présentées. Là où nous avions eu peur d'aller trop loin, nous étions en fait restés en-deça des possibilités du texte. Mais comment déterminer jusqu'où peut aller un comédien français, séparé du texte par plus de quarante ans et vingt mille kilomètres ? Toujours est-il que pour nous, traducteurs, cela nous a permis de voir jusqu'où va notre travail au service du texte, et le seuil au-delà duquel c'est le metteur en scène, ou le comédien, qui prend le relais. Notre expérience de travail avec une troupe nous l'a confirmé : autant nous pouvons réfléchir à des ajustements du texte, autant il est impératif que nous défendions nos principes. La traductologie est au service de la scénologie, dans une confrontation toujours enrichissante, mais jamais au prix d'un reniement de ses principes. Dans ce cas de collaboration, le traducteur se retrouve un peu dans la position d'un Dramaturg à l'allemande, passeur de culture sollicité, garant d'une certaine cohérence, mais aussi gardien du temple, en l'occurrence le texte(22).

En ce sens, la méconnaissance de la langue et de la culture coréenne est un atout pour nous, rendant le travail de collaboration très enrichissant, le revers de la médaille étant l'extrême difficulté d'intéresser des troupes à ces projets ! Ce pourquoi, dans les faits, nous travaillons beaucoup plus dans l'autre sens, en accueillant des troupes ou des chanteuses coréennes, pour lesquelles nous effectuons la traduction et le surtitrage. Dans les deux cas, c'est l'épreuve de l'oralité et l'apprentissage de l'humilité face au travail de troupe qui enrichit notre pratique. Mais il n'en reste pas moins qu'en tant que traducteur d'un texte de théâtre destiné à la lecture et à l'édition, on en revient toujours là : il faut prendre des décisions.

La spécificité du théâtre, c'est qu'il n'y a pas de notes de bas de page : voilà ce qui nous sert parfois à définir la spécificité de la traduction théâtrale, et souligne le fait que l'on a peu d'artifices, dans le texte même, pour faire passer les "Notions " sans les fausser, ni dans un sens (annexionnisme) ni dans l'autre (exotisme). Nous interdire les notes en bas de page nous a amenés à développer au mieux le paratexte, notes générales, préfaces, postfaces, de manière à répondre aux attentes légitimes d'un lecteur confronté à des œuvres dont il ignore tout, mais en préservant au mieux son approche de parasitages divers. Par exemple, pour une pièce dont deux protagonistes importants passent leur temps à parler de plats et d'alcools, nous avons choisi de conserver les termes coréens récurrents tels quels, et de mettre au début une " note gastronomique " regroupant les termes et expliquant de quoi il retournait. De même, dans À la soupe (déjà cité), l'argent étant un des moteurs de l'action, nous avons présenté hors

(22) Nous avons ainsi pu travailler avec la metteuse en scène coréenne Shin Meran, qui a monté en français Le train pour Séoul de Roh Kyeong-shik, avec des comédiens français, anglais et canadiens (Roh, 2004). 
texte l'équivalence approximative des wòns avec les francs de l'époque, et un récapitulatif des valeurs relatives entre elles des sommes en jeu dans le texte. Dans le cas de Muldoridong, nous avons été confrontés à un texte contemporain se référant en permanence à la tradition des danses masquées, jusque dans les noms des personnages : seul un important travail de préface peut rendre compte de cet aspect, mais cela n'empêche pas le texte de se nourrir de cette tradition, dans sa verve et sa poésie. Les titres et fonctions posent souvent problème, lorsqu'ils reviennent dans les dialogues, et les solutions adoptées peuvent varier : lorsque le héros vantard et ridicule d'une pièce, un certain Maeng, est dit jinsa pour avoir acheté un petit titre de ce nom, nous l'avons appelé Monsieur Maeng ; là où dans une autre pièce du même auteur, il est question du rêve d'accéder à l'aristocratie d'État que représente le titre très particulier de yangban, mot dont le héros se gargarise, nous avons conservé le mot coréen(23). Si des personnages ont des dénominations caricaturales, ou des surnoms évidents, il y a un certain plaisir à les traduire. Dans une pièce historique dont les deux protagonistes principaux sont des historiographes aussi cocasses que tragiques, clowns lunaires aux noms-portraits à charge moquant leur physique, et par lesquels ils s'interpellent tout au long de la pièce, nous n'avons pas hésité à les nommer Storiographe Grandguibolle et Storiographe Bobosse(24). Muldoridong nous posait un autre type de problème, avec des figures masquées archétypales dont le nom était une métaphore expressive, tantôt traduisible (par ex. l'Échalas), tantôt non. Et même si les Coréens savent que ces noms désignent "le Fâcheux " ou "le Simplet ", nous avons conservé leur nom propre coréen de Choraeng'i ou d'Imæ, tout en nous en expliquant dans la préface. Quant à la question de l'incapacité du lecteur français à prononcer (à haute voix ou intérieurement) les noms coréens, nous lui donnons une petite grille au début, en précisant qu'au bout du compte, c'est " au lecteur de s'inventer sa musique du coréen en français. "Et quant à l'hypothétique réalisation par des acteurs français de phrases contenant des mots coréens aussi étranges pour eux que "Dame Yonggyu de Tangsànwon ", "Viens ici, Ch'oraengi ! ", ou " Kùn-chol portait la petite Kùn-young sur son dos et courait vers Namwòn, dans le Cholla, au pied des monts Chiri ", il s'agit là d'un travail nécessaire, dont nous avons pu expérimenter qu'il était raisonnablement faisable, avec un peu d'effort.

\section{Le mot, la phrase, le phrasé}

Lorsque nous parlons de traduction de théâtre, nous nous apercevons que, souvent, l'arbre cache la forêt, c'est-à-dire que le mot cache la phrase dans lequel il est inséré. C'est assez clair dans tous les exemples dont nous venons de parler. Ce serait encore plus vrai avec les tra-

(23) 0 Young-jin, Monsieur Maeng marie sa fille (éd. LGR 2000), Du grand bonheur d'être Yangban (inédit).

(24) Roh Kyeong-shik, 2004, Le Souffle des siècles. 
ductions de proverbes, d'expressions toutes faites, de références, que l'on peut choisir de faire ressortir ou de faire oublier, et toute solution entre les deux. Un exemple simple : Monsieur Maeng rentre chez lui : il n'y a personne pour l'attendre. Scandalisé, il constate en bon coréen qu'il n'y " pas une souris ", là où le français attend " pas un chat ". Les traductologues peuvent en parler pendant des heures, mais le traducteur doit faire son choix. Un peu anecdotique dans le cas précédent, le problème devient plus sérieux lorsque Monsieur Maeng se prétend aussi rusé que Chegaryang, qui ne dit strictement rien au lecteur français ; mais se retrouvera-t-il pour autant aussi rusé qu'Ulysse ? Qu'un singe, qu'un Sioux ? Nous passons ici d'un choix de mot à son implication dans le domaine des "notions " au sens de Cullioli, et la responsabilité du traducteur est de prendre garde aux tentations d'annexions culturelles, surtout gréco-romaine ou judéo-chrétienne, si enfouies dans la langue parlée qu'on trouve ici ou là, au gré des traductions, des Coréens " forts comme Hercule ", ou proférant des "Mon Dieu ! " hors contexte. Si le choix de tel mot au lieu de tel autre présente un enjeu interculturel fort, c'est trop souvent à cette seule question que le débat se limite, au risque d'oublier l'essentiel, à savoir qu'un mot n'a de sens que pris dans une phrase. Ce sont les phrases qui donneront - ou pas - vie à tous ces mots problématiques, pris avec d'autres, enchâssés, charriés, et ces phrases elles-mêmes n'existent qu'en relation les unes avec les autres, dans une unité de type organique : le texte. Le traducteur est sans cesse confronté à des problèmes locaux, mots, expressions, proverbes etc., comme autant de pièces d'un puzzle énigmatique dont la solution ne peut être envisagée qu'à l'échelle globale du texte, là où se jouent les questions notionnelles, où s'organisent les choix entre le texte et le paratexte, où se construit le havre où accueillir le texte étranger, c'est-à-dire où s'invente son édition comme horizon (qu'il y ait ou non publication effective).

Le texte ainsi considéré, abouti, cadré, s'adresse hypothétiquement aussi bien à un lecteur qu'à un spectateur, et la boucle se boucle autour de cette notion d'oralité (intérieure ou effective) qui définit le théâtre, et constitue l'enjeu final de la traduction : si le mot est pris dans la phrase, les phrases sont prises dans un phrasé. Et ce phrasé, c'est celui du créateur, ce qu'on peut appeler une certaine manière de respirer, d'enchaîner les phrases. Si le traducteur reste trop à l'extérieur de ce geste créateur, on a l'impression, non tant qu'il ne traduit pas bien, mais surtout qu'il ne joue pas bien. Comme ces comédiens, même brillants, qui peinent à trouver la vitesse du texte, sa petite musique, sa respiration. D'une certaine manière, le traducteur peut même être considéré comme metteur en scène du texte(25). Nous écrivions, à propos de notre première traduction : "Le phrasé, c'est le texte lui-même, dans son mouvement d'écriture, avec ses vitesses différentielles, ses accélérations, ses enchaînements, ses bizarreries. Nous sommes persuadés, en ce qui concerne les textes ici traduits, qu'il

(25) Cf. Antoine Vitez : "Pour moi, traduction ou mise en scène, c'est le même travail, c'est l'art du choix dans la hiérarchie des signes ", cité in Henri Meschonic 1999, p. 398. 
n'y a pas besoin d'autres effets que ceux qui sont présents dans le phrasé d'0 Young-jin pour manifester son étrangeté. Si cette étrangeté est coréenne, elle nous importe comme étant partie intégrante de son écriture, et à phraser de l'intérieur, le plus possible de l'intérieur du texte(26). "

\section{Conclusion}

Nous avons vite appris que les éditeurs spécialisés dans la littérature coréenne ne voulaient pas de théâtre, et que les éditeurs spécialisés dans le théâtre ne voulaient pas de coréen... Aussi la venue en France de la pièce Muldoridong en 2004 nous a littéralement placés "à la courbe des eaux ", et nous avons franchi le pont. Nous avons compris combien notre travail de traducteur pouvait gagner à se retrouver au centre d'un dispositif tourné vers la diffusion (collection chez Imago, surtitrage, appareillage critique, lien avec l'université, implication des Fondations). Aider à la réception du texte traduit, en lui donnant la chance d'aller à la rencontre du lecteur, du spectateur : pour tout cela, l'expérience de la traduction théâtrale est irremplaçable.

ymhanpej@gmail.com

HAN Yumi est née à Séoul. Elle est titulaire d'un doctorat en linguistique de l'Université Paris 7 sur le bilinguisme coréen-français (2001) et achève actuellement une deuxième thèse sur le pansori en Études Coréennes à l'Université Paris 7 (soutenance prévue en décembre 2010). Enseignante en études coréennes à l'Université de La Rochelle et à l'Université Paris 7 (2007-2009), elle est membre du Centre de la Recherche sur la Corée (CRC), UMR 8173 Chine-Corée-Japon CNRS-PARIS 7 et est également chercheuse invitée à l'Université Nationale de Séoul, au Centre international sur les Études Coréennes (Séoul, Kyuganggak Junior Fellow, 2010). Elle est co-directrice avec Hervé Péjaudier de la collection "Scènes Coréennes " aux Éditions Imago (depuis 2004). En tant que traductrice indépendante, elle a été Lauréate de la Fondation Daesan $(1998,2000,2006)$ et de l'Institut Coréen pour la Traduction Littéraire $(2004,2006)$ à Séoul et a reçu le Prix culturel France-Corée en 2000 (pour la connaissance du théâtre coréen en France). Spécialisée dans les arts de la scène coréens anciens et modernes, elle a réalisé en particulier les surtitrages des pansori en France (Festival d'Automne, Festival de l'Imaginaire, Musée du Quai Branly et Musée Guimet).

www.editions-imago.fr/IMAGO/menulitteraturecoree

(26) Han Yumi et Hervé Péjaudier, "Quelques remarques... ", in 0 Young-jin, Monsieur Maeng marie sa fille, op. cit. 


\section{Bibliographie}

BERMAN Antoine, 1984, L'épreuve de l'étranger, Paris, Gallimard.

CHOE Junho, 2005, "Le théâtre coréen d'hier à aujourd'hui, et ses échanges avec la France ", in Culture Coréenne, $n^{\circ}$ 70, p. 2-11, consultable sur le site www.coree-culture.org

CULIOLI Antoine, 2002, Variations sur la linguistique, Paris, Klincksieck.

ECO Umberto, 2003, Dire presque la même chose, Paris, Grasset.

HAN Yumi et PÉJAUDIER Hervé, 2007, "La question de l'étrangeté en traduction littéraire (Coréen-Français) ", p.155-170, Munich, Multilinguale Kommunikation, Translinguae 2, Éditions Martin Meidenbauer.

HO Kyu, 2004, Muldoridong, le village à la courbe des eaux, trad. HAN Yumi et Hervé PÉJAUDIER, Paris, Imago, coll. "Scènes Coréennes ".

LEDERER Marianne, 1994, La traduction aujourd'hui - le modèle interprétatif, Paris, Hachette.

LEE Gun-sam, 2010, À la soupe ! trad. Kim Hyon-Sook, revue par HAN Yumi et Hervé PÉJAUDIER, postface de CHOE Junho, Paris, Imago, coll. "Scènes Coréennes ".

MESCHONNIC Henri, 1999, Poétique du traduire, Lagrasse, Verdier.

0 Young-jin, 2000, Monsieur Maeng marie sa fille, trad. HAN Yumi et Hervé PÉJAUDIER, Paris, éd. Librairie Galerie Racine.

RAPIN Cathy et IM Hye-Gyeong, 2006, Théâtre coréen, anthologie, Paris, éd. Imago, coll. "Scènes Coréennes ".

RAPIN Cathy et IM Hye-Gyeong, 2006, Théâtre coréen d'hier et d'aujourd'hui, Paris, éd. de l'Amandier 2006.

RICOEUR Paul, 2004, Sur la traduction, Paris, Bayard.

ROH Kyeong-shik, 2004, Un pays aussi lointain que le ciel, suivi de Le train pour Séoul et Le souffle des siècles, trad. HAN Yumi et Hervé PÉJAUDIER, Paris, Imago, coll. "Scènes Coréennes ". 